\title{
Compact fibre Bragg grating-based thermometer for on-line temperature monitoring of drill bits
}

\author{
Kenny Hey Tow ${ }^{\mathrm{a},}$, Miguel Llera ${ }^{\mathrm{b}}$, Sébastien Le Floch ${ }^{\mathrm{b}}$, Yves Salvadéb and Luc Thévenaz ${ }^{\mathrm{a}}$ \\ ${ }^{a}$ EPFL Swiss Federal Institute of Technology, Group for Fibre Optics, SCI-STI-LT, Station 11, 1015 \\ Lausanne, Switzerland; \\ ${ }^{\text {b }}$ Haute Ecole Arc Ingénierie, Groupe de compétence en métrologie et vision industrielle, Rue de la \\ Serre 7, 2610 Saint Imier, Switzerland.
}

\begin{abstract}
In this communication, a novel compact fibre Bragg grating-based thermometer for on-line temperature monitoring of drill bits is reported. Our proposed technique can potentially be used to optimize any drilling process, requiring the use of small drill bits, through direct temperature measurement at the drill bit instead of relying on indirect parameters (speed of rotation, applied force) in order to avoid an overheating as it is currently done nowadays.
\end{abstract}

Keywords: Temperature monitoring, drill bit, synchronous detection, fibre Bragg grating

\section{INTRODUCTION}

Drilling is nowadays a widely used operation, whether it is to make holes of various sizes and depths for the manufacturing of mechanical and electrical components or for modern surgery procedures to drill bones in orthopedics/traumatology and dentistry. During the drilling process, most of the mechanical energy is converted into frictional heat. This temperature rise may result in damages such as rapid tool wear and diametrical errors in hole size during mechanical processes, drill smear $^{1}$, which affects the reliability of printed circuit boards (PCBs), or irreversible osteonecrosis if the temperature is above $47^{\circ} \mathrm{C}$ during bone drilling ${ }^{2}$. To reduce these aforementioned risks due to over-heating, thermocouples ${ }^{3}$, infra-red thermometers ${ }^{4}$ and infra-red cameras ${ }^{1}$ have been used for temperature monitoring of drill bits (Table 1). Thermocouples and infra-red (IR) thermometers are easy to use, but are not particularly suited for applications requiring the use of small drill bits, for instance during bone drilling for medical surgery or hole drilling in PCBs. The latter do not give accurate measurements for small objects and thermocouples are challenging to integrate inside small drill bits. Moreover, continuous measurements are difficult to achieve with these devises. On the other hand, IR cameras offer the advantage of providing accurate, real-time temperature monitoring, but these systems are expensive, require additional material (macrolens and temperature field image) for temperature monitoring of small drill bits and only information on the temperature at the cut surface can be retrieved.

Table 1. Comparison of different devices, which can be used for temperature monitoring of drill bits.

Marks are from very positive $(++)$ to very negative $(--)$.

\begin{tabular}{|c|c|c|c|c|}
\hline & \multicolumn{4}{|c|}{ Device used for temperature monitoring of drill bits } \\
\hline Parameters & Thermocouple & $\begin{array}{c}\text { IR } \\
\text { thermometer }\end{array}$ & $\begin{array}{c}\text { IR } \\
\text { camera }\end{array}$ & $\begin{array}{c}\text { Fibre Bragg } \\
\text { grating }\end{array}$ \\
\hline Accuracy & + & + & ++ & ++ \\
\hline Suitability for small drill bits & -- & -- & - & ++ \\
\hline Continuous measurement & -- & -- & + & + \\
\hline Cost of the monitoring system & ++ & ++ & -- & - \\
\hline
\end{tabular}

*Further author information: send correspondence to Kenny Hey Tow (kenny.heytow@epfl.ch)

Sixth European Workshop on Optical Fibre Sensors, edited by Elfed Lewis, Proc. of SPIE Vol. 9916, $99162 \mathrm{~F} \cdot$ (C) 2016 SPIE · CCC code: 0277-786X/16/\$18 · doi: 10.1117/12.2235871 
Fibre Bragg Gratings (FBGs) have not yet been used for temperature monitoring of drill bits even though they are simple, miniature (a few mms long and 1/10 of $\mathrm{mm}$ in diameter) sensing elements encompassing all the advantages attributed to optical fibres (electrically insulating, chemically inert, etc.) and are sensitive to temperature changes ${ }^{5}$, which make them very good candidates for temperature monitoring of small drill bits. However, it requires a customized drill bit in which the FBG is inserted and disposed once the drilling tool is no longer usable.

\section{EFFICIENT LIGHT COUPLING INSIDE A ROTATING DRILL BIT}

Since the FBG will be located inside the rotating drill bit, a free space optical coupling between the rotating FBG and a fixed lead fibre is required to bring incident light to the FBG and route the back-reflected light, in which the useful information on temperature change is encoded, to the interrogation/demodulation system (Figure 1). Direct fibre coupling, without the use of any optical interface, can be used, with very little efficiency though since coupling losses will inevitably arise due to misalignment between the two fibres due to mechanical instabilities and mechanical clearance between the drill fixture and the mandrel. Moreover, dust and debris produced during the drilling process and the presence of fluids (if irrigation is needed during drilling) will also

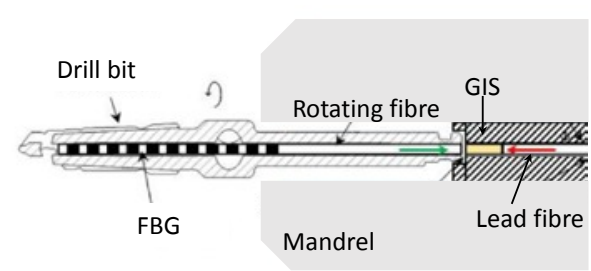

Figure 1. Proposed free space optical coupling system between a rotating and a fixed fibre.

Red arrow: path of incoming light; Green arrow: path of the reflected light from the FBG contribute in lowering the signal to noise ratio of the collected light. In such configurations, fibre collimators with lenses at the fibre tip are usually used to ensure robust optical coupling, but they are not compact enough to be integrated inside mandrels making use of small drill bits. Alternatively, more compact solutions such as fibre-based collimators with gradedindex fibre tips ${ }^{6}$ (GI) and graded-index fibre tips with optical spacers ${ }^{7}$ (GIS), can be used. Preliminary simulations were performed on Zemax ${ }^{\circledR}$ to determine the optimal lengths for the fibred collimators and two fibred collimators were made. For the GI configuration, the built graded-index portion lengths were of 262 and $263 \mu \mathrm{m}$ and for GIS configuration, the produced tips had spacer lengths of $149 \mu \mathrm{m}$ and $159 \mu \mathrm{m}$ and graded-index lengths of $149 \mu \mathrm{m}$ and $132 \mu \mathrm{m}$ respectively. The misalignments losses on a roundtrip due to longitudinal and transverse offsets as well as tilt were measured for both configurations and plotted on Figure 2 and are compared to predicted losses (red plots) obtained using Zemax®.
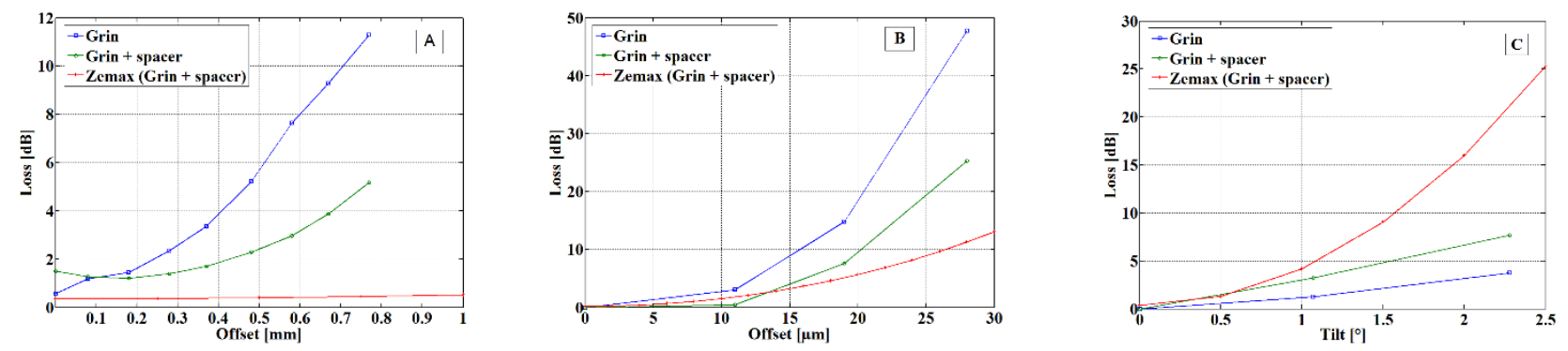

Figure 2. Misalignment losses measured for the two configurations: graded-index (GI), graded-index with spacer (GIS), and Zemax ${ }^{\circledR}$ simulation (Zemax) for longitudinal offset (A), transverse offset (B), and tilt (C).

The GIS configuration was chosen for our system since it proved to be more robust to offsets and tilts, which arise during the drilling process. Transverse offset and tilt being very critical for overall coupling losses, a quartz ferule was used to decrease the possible transverse offset to about $3 \mu \mathrm{m}$, while also avoiding any possible tilt.

\section{INTERROGATION SYSTEM BASED ON SYNCHRONOUS DETECTION AND WAVELENGTH TRACKING}

The experimental setup for our interrogation system is schematically represented in Figure 3. Light from a tunable DFB laser is sent to the FBG, integrated inside a rotating drill bit, using a circulator. The reflected light is collected and sent to a photodector for analysis. In our interrogation system, the reflected signal from the FBG can be used in a similar way as an atomic/molecular transition for wavelength modulation spectroscopy ${ }^{8}$, a widely employed technique to produce derivative-like signals of an absorption line that are used for laser stabilisation to the centre (using the first - $1 f$ - or third $3 f$ - harmonic signal) or edge (using the second -2f- harmonic signal) of a frequency discriminator. 


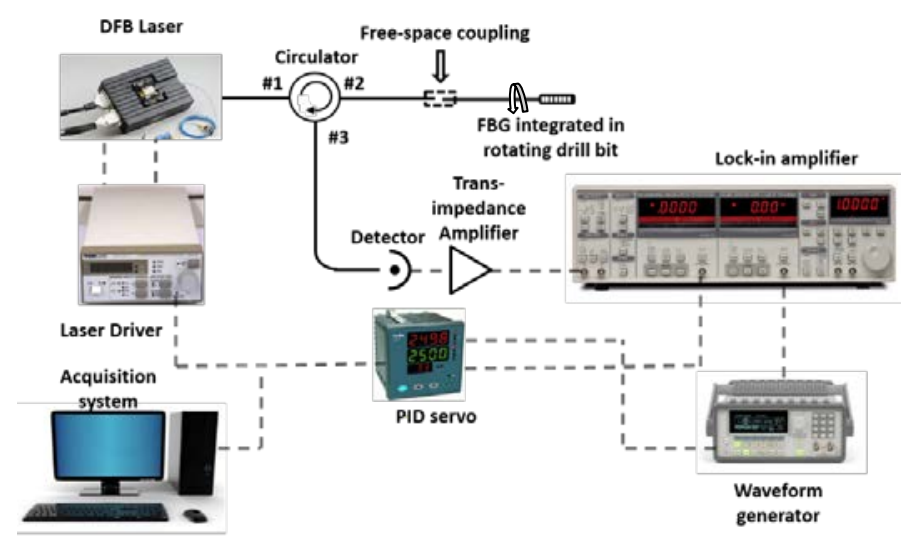

Figure 3. Experimental setup used to monitor the temperature change at the drill bit.

The wavelength dithering is performed by applying a small periodic signal at a frequency of $16.67 \mathrm{kHz}$ to the DFB laser's current. This dithering frequency is chosen to be much larger than the typical rotational speed of $3000 \mathrm{tr} / \mathrm{min}$ of drills so that the lock-in detection does not "see" the reflected light as an amplitude-modulated light. When the laser is tuned on the edge of the FBG, this wavelength modulation produces a modulation of the reflected optical power. This intensity modulation contains several harmonics of the modulation frequency and a lock-in detection is used to extract the first harmonic, the error signal $\varepsilon$. Any change in temperature of the drill bit will result in a shift of the FBG's central wavelength. The measured value of $\varepsilon$ is sent to a Proportional Integral Derivative (PID) servo, which provides a correction voltage to the laser driver; thereby locking the laser frequency to the zero crossing point of the error signal, which corresponds to the Bragg wavelength of the FBG. The measurement of this correcting voltage, which varies linearly with the wavelength shift, directly gives the change in temperature triggering it.

\section{PROOF-OF-CONCEPT OF THE FBG-BASED TEMPERATURE MONITORING SYSTEM PERFORMED OF A DRILL BIT}

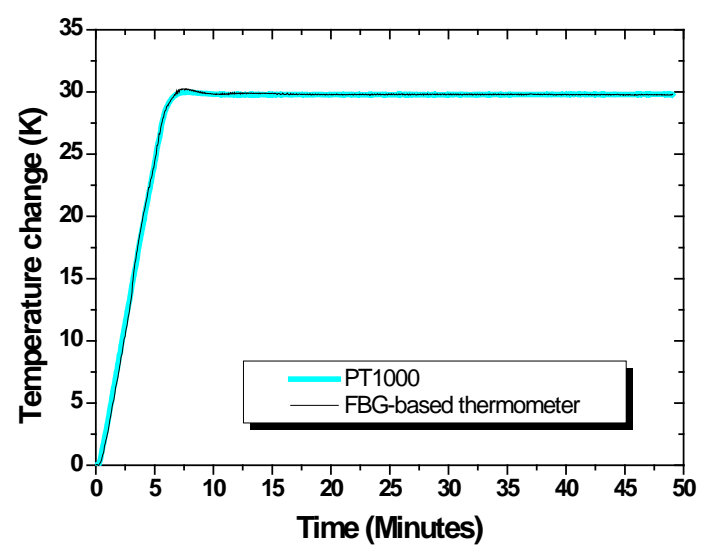

(A)

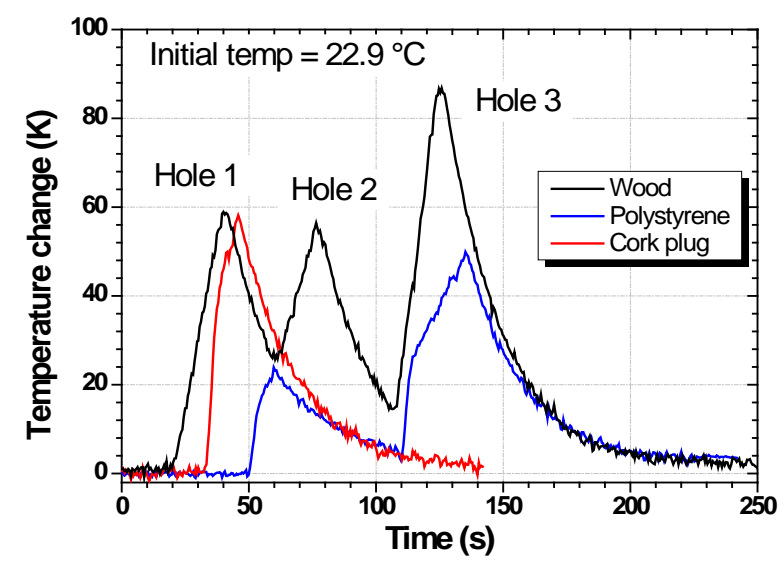

(B)

Figure 4. (A) Temporal evolution of the temperature change of a heated water bath simultaneously measured at the drill proposed FBG-based temperature monitoring system and a PT1000 thermometer. (B) Temperature change measure demonstrator at the drill bit/material interface during a drilling test performed in wood, cork plug and polystyrene.

The validation tests for our FBG-based temperature monitoring system was performed using a modified mandrel, as shown in Figure 1. A FBG was inserted in the middle of a drill bit and the contactless optical coupling system, described in Section 2, was used to both inject incident light and collect the reflected light from the FBG. The drill bit of the modified mandrel was placed in a heated water bath, next to a PT1000 thermometer, and made to rotate. The change in temperature of the 
water bath, measured both by our proposed system match the measurements obtained by the PT1000 sensor as shown in Figure 4A, which validates our interrogation system. Several tests involving drilling holes into different materials while performing on-line temperature monitoring at the drill bit-material interface were also performed and examples of temperature monitoring while drilling in wood, cork plug and polystyrene are plotted on Figure 4B. With our current demonstrator, temperature changes of $>50 \mathrm{~K}$ from the initial temperature with a resolution of $<1 \mathrm{~K}$ can be measured. The measurement range can be easily extended by, for instance, using another DFB laser with more wavelength tuneability.

A standard deviation of around $0.4 \mathrm{~K}$ was calculated for these measurements, performed with a $100 \mu$ s time constant for the lock-in detection. This relatively high value is due to the interferometric noise brought by the varying mechanical clearance between the drill bit and the mandrel. The measurements can be made more precise by either using a longer time constant to attenuate more this noise since fast refresh rates are not required for this application (typically $0.5 \mathrm{~s}$ ) or by making the contactless coupling system more robust to interferometric noise (anti-reflection coating, reduction of the mechanical clearance, etc.)

\section{CONCLUSION}

In this communication, a novel fibre Bragg grating-based thermometer for real-time temperature monitoring of drill bits is proposed. The proof-of-concept of our system was demonstrated for improved temperature management during drilling. Temperature changes of $>50 \mathrm{~K}$ from the initial temperature with a standard deviation of $0.4 \mathrm{~K}$ and at a refresh rate of $<<$ $0.5 \mathrm{~s}$ can easily be measured. Our proposed system can be implemented for many applications requiring temperature monitoring during drilling, ranging from hole piercing to ensure more reliability in PCBs to bone drilling during surgery

to help deliver a safer outcome for medical care, with a much reduced probability of harming aftermath through reduced suffering for the patient.

\section{ACKNOWLEDGEMENTS}

The authors would like to acknowledge Rüeger SA (Switzerland) and the Swiss Commission for Technology and Innovation (Project n 17046.1) for their support.

\section{REFERENCES}

[1] Fu, L. and Guo, Q., “On-line temperature measurement of a micro drill bit,” Circuit World 38, 55-58 (2012).

[2] Augustin, G., Davila, S., Mihoci, K., Udiljak, T., Vedrina, D. S. and Antabak, A., "Thermal osteonecrosis and bone drilling parameters revisited,” Archives of Orthopaedic and Trauma Surgery 128, 71-77 (2008).

[3] Bogovic, V., Svete, A., Rupnik, K., and Bajsic, I., "Experimental analysis of the temperature rise during the simulation of an implant drilling process using experimental designs," Measurement 63, 221-231 (2015).

[4] Traub, A. and Evans, J., "Thermal monitoring of drill bits during laminate drilling," Circuit World 13, 37-41 (1987).

[5] Kersey, A. D., Davis, M. A., Patrick, H. J., LeBlanc, M., Koo, K., Askins, C., Putnam, M. and Friebele, E. J., "Fiber grating sensors,” Journal of Lightwave Technology 15, 1442-1463 (1997).

[6] Emkey, W. L., Jack, C., “Analysis and evaluation of graded-index fiber lenses,” Journal of Lightwave Technology 5, 1156-1164, (1987).

[7] Mao, Y. Chang, S., Sherif, S., and Flueraru, C., "Graded-index fiber lens proposed for ultrasmall probes used in biomedical imaging,” Applied Optics 46, 5887-5894 (2007).

[8] Supplee, J. M., Whittaker, E.A., and Lenth, W., "Theoretical description of frequency modulation and wavelength modulation spectroscopy,” Applied Optics 33, 6294-6302, (1994). 